\title{
Gradientes de empleo intrarregionales en Colombia*
}

\author{
Julián Durán Peralta,' Carlos Federico Vallejo Mondragón² \\ Universidad Santiago de Cali (Colombia)
}

Recibido: enero 30 de 2015 - Revisado: marzo 3 de 2015 - Aceptado: mayo 15 de 2015

Referencia formato APA: Duran-Peralta, J., \& Vallejo, C. F. (2015). Gradientes de empleo intrarregionales en Colombia. Revista Cientifica Guillermo de Ockham, 13(1), 117-124.

\section{Resumen}

Se calcula la tasa (o gradiente) a la que el empleo desciende conforme aumenta la distancia entre las capitales regionales y los municipios periféricos en Colombia, en el año 2005. El empleo en el país se localiza en la parte central, en el sector andino montañoso y en el Caribe. Por lo tanto, se analiza un conjunto de las siete regiones más representativas del país por su nivel de actividad económica. Para medir el gradiente de empleo, se estima un modelo log-lin por M.C.O. y se utiliza la información de empleo del censo DANE del 2005. Los resultados muestran que en cinco de las siete regiones el gradiente es estadísticamente significativo y con signo negativo, por lo que el empleo se reduce a partir de las capitales hacia los municipios distantes. En consecuencia, este cálculo permite establecer en qué regiones el empleo se concentra más en torno a la capital regional de acuerdo con el patrón sugerido en la ley de Clarck, lo que da pie a elementos por considerar en la política de desarrollo regional.

Palabras clave: Localización, gradientes de densidad de empleo, economía espacial

\section{Intraregional employment gradients in Colombia}

\section{Abstract}

We calculate the rate (or gradient) at which employment declines depending on the increase in the distance between regional capitals and peripheral municipalities in Colombia in 2005. Employment in Colombia is concentrated in the central region, in the mountainous andean sector and the Caribbean, therefore, a set of seven regions, which are the most representative in the country because of their economic activity, were analyzed. In order to measure the rate, a log-lin model by OLS is estimated using employment information from the DANE Census of 2005. The results show that in five of the seven regions, the gradient is statistically significant and negative, because employment is reduced from the capital to distant municipalities, following the pattern of Clarck's law, leading to items to be considered in regional development policy.

Keywords: Location, employment density gradients, spatial economics

\footnotetext{
* Este artículo se deriva del proyecto de investigación Tendencias en el mercado laboral colombiano, el autoempleo y sus determinantes microeconómicos y de entorno regional.

1. MSc en economía aplicada, grupo de investigación en desarrollo económico y economía internacional. Dirección postal Calle 5 No. 62-00 Bloque 7 piso 6 Campus Pampalinda Cali-Colombia. E-mail: julian.duran02@usc.edu.co

2. MSc en políticas públicas, grupo de investigación en desarrollo económico y economía internacional, E-mail: federico.vallejo00@usc.edu.co
} 


\section{Intrarregional gradientes emprego na Colômbia}

\section{Resumo}

É calculado Taxa (ou gradiente) em que o emprego diminui com o aumento da distância entre as capitais regionais e municípios periféricos na Colômbia em 2005. O emprego no país está localizado na parte central, no setor andino montanhosa é calculada e no Caribe, portanto, um conjunto de sete regiốes que são os mais representativos do país para o seu nível de atividade econômica é analisada. Para medir o uso de gradiente modelo log-lin é estimado por OLS e informaçáo sobre o emprego DANE Censo 2005. Os resultados mostram que em cinco das sete regióes do gradiente é estatisticamente significativa e negativa, de modo que o emprego é reduzida a partir da capital aos municípios remotos é usado. Consequentemente, esse cálculo permite determinar em que regióes o emprego está mais concentrado em torno da capital regional, seguindo o padrão direito Clark sugeriu, dando origem a elementos a considerar na política de desenvolvimento regional.

Palavras Chave: Localização, gradientes de densidade de emprego, economia espacial

\section{Introducción}

Colombia es un país cuya actividad económica se ha concentrado en la parte central en torno a su capital Bogotá, en la región andina montañosa y en la región de la costa caribe. Además, la geografía del país está separada por tres cordones de cordilleras, lo que ha implicado que su economía se haya desarrollado a partir de mercados fragmentados en regiones. Históricamente, cada región ha tenido su dinámica propia, sus mercados y estructuras productivas se han formado con cierto grado de autonomía (Ocampo, 1986). Así, un aspecto que resulta relevante es establecer cómo se ha distribuido el empleo a nivel de cada región y si esa distribución sigue los patrones que Clark (1951) sugiere en su economía espacial. Según Clark, el empleo desciende de forma exponencial desde el centro económico regional hacia los asentamientos periféricos. Este análisis contribuirá a establecer las regiones donde el empleo se concentra más en torno a la ciudad capital o donde tiende a estar disperso, elemento a tener en cuenta en las políticas de desarrollo regional.

Al momento de abordar el estudio de la distribución del empleo través de las ciudades o las regiones, una herramienta de análisis útil es el cálculo de los gradientes de densidad, el cual está enmarcado dentro de los modelos de la economía espacial de Clark. En esta materia, se destacan internacionalmente investigaciones que han medido y analizado tanto de forma estática como dinámica, los gradientes de empleo o población en importantes ciudades del mundo (Gordon, Richardson \& Wong, 1986; McDonald, 1989; Mukhopadhyay \& Dutt, 1994; Martori \& Surinach, 2002; Glaeser \& Kahn, 2001; Bunting, Filion
$\&$ Priston, 2002; Coffey \& Shearmur, 2002; Millward \& Bunting, 2008; Lu \& Guldmann, 2012).

También son variadas las aproximaciones metodológicas para enfrentar esta problemática. Así, Mcmillen (2004), analiza un conjunto de ciudades norteamericanas aplicando modelos de autocorrelación espacial, mientras que otros trabajos estiman funciones cubic spline (Anderson, 1982; Craig \& Ng, 2001; Muñiz, Galindo \& García, 2003). Diversos estudios se han enfocado en la estimación de gradientes como una forma de identificar subcentros económicos, (Gordon et al., 1986; McDonald, 1987; McDonald \& Prather, 1994; Craig \& Ng, 2001; Mcmillen \& Lester, 2003; Redfearn, 2007; Veneri, 2013).

En Colombia, el estudio de gradientes de empleo ha sido escaso, entre ellos los estudios de Avendaño (2008) y Ruiz, Roca \& Moix (2012) que miden los gradientes de empleo para la ciudad de Bogotá centrados en la búsqueda de subcentros económicos.

En el presente estudio se calculan gradientes del empleo en el interior de las regiones de Colombia durante el 2005. Se parte de un enfoque regional, pues si bien los trabajos internacionales se centran en la identificación de subcentros o de un policentrismo metropolitano, al abordar el problema para los centros que agrupan la actividad económica en Colombia, por su dinámica histórica cabe suponer que estos se configuran más como centros independientes con un gradiente individual (a la Clarck) que como un esquema a la Cristaller caracterizado por un esquema de hexágonos concéntricos al Central park y subcentros periféricos. Para ello, se estima un modelo log-lin por M.C.O. que mide la tasa a la que el empleo desciende 
desde las capitales regionales hasta los municipios periféricos. De esta forma, las unidades de observación son los municipios. Los resultados muestran que en cinco de las siete regiones analizadas el gradiente es estadísticamente significativo y con signo negativo.

\section{Fundamentos teóricos}

La relación formal entre la densidad de población y su distribución espacial fue planteada inicialmente por Clark (1951), en lo que ha sido llamado como la ley de Clark. Basado en la evidencia empírica, este autor planteó que la densidad de la población o del empleo en un área urbana tiende a caer de forma exponencial desde el centro hacia la periferia. Así, trata de formalizar a través de una sencilla ecuación una relación que obedece a ciertas regularidades empíricas.

No obstante, dicha relación empírica también podría aparecer en las regiones, por lo que es viable establecer que las ciudades y poblaciones en una región determinada siguen ese patrón de distribución. En consecuencia, puede definirse $Y$ como la densidad de empleo en un punto $i$ del espacio y $X$ la distancia entre el punto $i$ y el centro económico. Entonces, se tendría:

$$
Y_{i} Y_{i}=b_{0} e^{-b_{1} X_{i}}(1)
$$

donde, $b_{0}$ es el nivel de empleo en el centro económico y $b_{1}$ es el gradiente de densidad de empleo, el cual representa la tasa a la que desciende el empleo del centro hacía la periferia.

Ahora bien, aunque Clark solo enfatiza en establecer una relación exponencial negativa, es posible sugerir un conjunto de argumentos teóricos que contribuyen a justificarla, y más en un contexto intrarregional.

De un lado, es claro que la localización de las firmas y los hogares tiene una relación de interdependencia, ya que los hogares buscan las firmas como fuentes de empleo y las firmas buscan a los hogares como consumidores. Así, en cada región existirá un emplazamiento que se impondrá como el centro económico y será el lugar donde se ubique el mayor volumen o densidad de empleo (Krugman, 1991). El centro económico constituye el lugar donde se obtienen las mayores economías de escala tanto internas como externas, en la producción y en el consumo, asociadas a la presencia de encadenamientos productivos, la existencia de mercados de trabajo especializados, los spillovers del conocimiento, la amplia oferta de bienes públicos y la oferta de servicios de consumo y entretenimiento que favorece el establecimiento de hogares, entre otros factores (Jacobs, 1975; Sveikauskas, Gowdy \& Funke, 1988; Krugman, 1991; Glaeser, Kallal, Scheinkman \& Shleifer, 1992; Henderson, Kuncoro \& Turner, 1995; Fujita \& Thisse, 1996; Audretsch \& Feldman, 1996). No obstante, a medida que haya un alejamiento del centro, se empezarán a perder esas economías de escala, pérdida que puede ser exponencial debido a la no linealidad que presentan esas economías. Por consiguiente, las decisiones de localización tanto de las firmas como de los hogares serían determinadas por la presencia de estas economías. Así, la configuración de una distribución espacial del empleo de forma decreciente obedece a ese tipo de racionalidad económica.

Un segundo argumento complementario del anterior, se refiere a la interacción entre la renta de localización y los costos de transporte. Si se parte del modelo de Von Thünen (1826) y Dunn (1954; 1955), cabe plantear que las actividades económicas se localizarán de acuerdo con su renta (derivada de la localización) por unidad de suelo. De esta manera, las actividades de mayor rentabilidad se ubicarán en el centro económico y a medida que se alejen de ese centro se reducirá la rentabilidad y se incrementarán los costos de transporte.

Sin embargo, diversos factores geográficos, administrativos e institucionales pueden afectar una configuración espacial de las actividades económicas de la región según la ley de Clark. Por ejemplo, si las ciudades o poblados están muy alejados del centro económico, los costos de transporte se hacen muy elevados y se favorece la aparición de dinámicas locales o subregionales autónomas o independientes del centro, mostrando así que la densidad de empleo podría aumentar en poblados lejanos, situación que Polése (1998) denomina efecto protector del espacio. Si los poblados son relativamente cercanos al centro pero tienen deficientes redes de transporte, el resultado es el mismo del punto anterior.

\section{Método}

\section{Datos}

La cuestión metodológica inicia con la identificación y el procesamiento de fuentes de información secundaria sobre el empleo y las distancias entre las ciudades y los municipios. Los datos del empleo fueron tomados del censo de población 2005 del DANE y la distancia (en kilómetros) de ruta entre la respectiva capital de departamento y los municipios, fue obtenida del portal web lasdistancias. 
com. Como segundo paso, se eligieron las principales regiones del país para ser tomadas en el análisis. En el censo poblacional, el DANE efectuó la siguiente clasificación de las regiones del país con los departamentos que las conforman: Pacífico Norte (Antioquia), Pacífico Sur (Valle del Cauca), Andina Norte (Santander y Norte de Santander), Andina Sur (Huila, Tolima, Cauca y Narińo), Central (Cundinamarca y Boyacá), Eje Cafetero (Caldas, Risaralda y Quindío), Caribe (Atlántico, Bolívar, Cesar, Córdoba, La Guajira, Magdalena y Sucre). Otras regiones fueron Amazonia, Orinoquia, Chocó y San Andrés y Providencia. Este último conjunto de regiones no fueron incluidas en el presente estudio debido a su menor peso en la actividad económica nacional. Así mismo, Bogotá D.C. se incluyó bajo el supuesto de ser capital del departamento de Cundinamarca.

\section{Resultados}

En la Tabla 1 se exponen las medias y medianas del empleo y la distancia, correspondientes a los municipios que integran cada región. Así, la región Pacífico Sur presenta el mayor promedio de empleo por municipio, con un valor de 32.824 ocupados y la mediana de 13.755 . En este caso, la mediana resulta una medida más apropiada del valor central del número de ocupados. Así, el eje cafetero y el Pacífico norte son las dos siguientes regiones que tienen valores más altos en la mediana del empleo con 10.605 y 10.306 ocupados, respectivamente. Por consiguiente, en las anteriores regiones los municipios tienden a tener mayores niveles de empleo respecto de los municipios de otras regiones. En cuanto a la distancia a la capital del departamento, se observa que las regiones Pacífico norte, Andina Norte y Caribe, registran las mayores distancias promedio de los municipios, mientras que las regiones Eje Cafetero y Central tienen los menores promedios, lo cual indica que en estas últimas hay una mayor concentración espacial de los municipios en torno a las capitales de los departamentos o una menor separación.

La Tabla 2 muestra que las regiones que tienen mayores niveles de ocupación son la Central, la cual incluye a Bogotá D.C., seguida de la región Caribe que agrupa un conjunto de siete departamentos. Posteriormente, está Pacífico Norte, constituida por Antioquia, departamento que alberga una gran cantidad de municipios. También existen regiones con baja densidad de empleo por kilómetro cuadrado, como es el caso de las regiones Caribe y Andina Sur y otras con alta densidad (Central, Pacífico Sur y Eje Cafetero).

Tabla 1

Estadísticas descriptivas de los municipios a través de las regiones

\begin{tabular}{|c|c|c|c|c|}
\hline \multirow[b]{2}{*}{ Región } & \multicolumn{2}{|c|}{ Ocupados ańo 2005} & \multicolumn{2}{|c|}{ Distancia a la capital del departamento ( $\mathrm{km}$ en ruta) } \\
\hline & Media & Mediana & Media & Mediana \\
\hline Pacífico Norte & 14.719 & 10.306 & 136 & 104 \\
\hline Pacífico Sur & 32.824 & 13.755 & 108 & 106 \\
\hline Andina Norte & 8.849 & 8.145 & 124 & 121 \\
\hline Andina Sur & 7.634 & 7.756 & 103 & 98 \\
\hline Central & 15.870 & 7.759 & 94 & 87 \\
\hline Eje Cafetero & 14.174 & 10.605 & 65 & 65 \\
\hline Caribe & 11.410 & 8.130 & 117 & 83 \\
\hline Total & 12.982 & 8.605 & 109 & 93 \\
\hline
\end{tabular}

Fuente: DANE. Censo de población 2005

Tabla 2

Ocupados y densidad de las regiones

\begin{tabular}{lccc}
\hline \multicolumn{1}{c}{ Región } & Ocupados & Extensión $\left.\mathbf{( k m}^{\mathbf{2}}\right)$ & ${\text { Ocupados } \mathbf{k} \mathbf{k m}^{\mathbf{2}}}$ \\
\hline Pacífico Norte & 1.839 .820 & 63.612 & 28,92 \\
\hline Pacífico Sur & 1.378 .607 & 22.195 & 62,11 \\
\hline Andina Norte & 1.061 .939 & 52.185 & 20,35 \\
\hline Andina Sur & 1.442 .832 & 105.580 & 13,67 \\
\hline Central & 3.808 .732 & 49.000 & 77,73 \\
\hline Eje Cafetero & 751.247 & 13.873 & 54,15 \\
\hline Caribe & 2.179 .359 & 131.628 & 16,56 \\
\hline Total & 12.462 .535 & 438.073 & 28,45 \\
\hline
\end{tabular}

Fuente: DANE. Censo de población 2005, Gobernaciones departamentales.

$120<$ Universidad de San Buenaventura, Cali - Colombia 
Finalmente, la ecuación (1) se estima a través de un modelo econométrico log-lin, cuyo parámetro $\beta_{1}$ representa el gradiente de densidad.

\section{Análisis de resultados}

Los resultados de la estimación de la ecuación (1) son presentados en la Tabla 3. El modelo se estimó con errores estándar robustos para corregir la presencia de heterocedasticidad.

Se aprecia que en todas las regiones los gradientes obtuvieron signos negativos, en concordancia con el modelo de Clark. Sin embargo, en las regiones Pacífico Norte y Andina Sur los gradientes no arrojaron significancia estadística.

Las regiones con los mayores valores fueron Pacífico Sur, con un gradiente de $-0,85 \%$ y eje cafetero con $-0,754 \%$. Esto indica que por cada kilómetro de distancia en ruta que se separe el municipio de la capital de departamento en la región Pacífico Sur, el empleo decrece en un $0,859 \%$ y por cada kilómetro de distancia a las capitales de los departamentos del eje cafetero, el empleo decrece en un $0,754 \%$. En estas regiones que sustentan los más altos gradientes, el empleo tiende a concentrarse más alrededor de las capitales, lo cual indica una gran dependencia o subordinación de las economías subregionales o municipales a la capital.

En las Figuras 1.a y 1.b se muestra que el empleo disminuye a medida que hay un alejamiento de las capitales, por lo que en estas regiones la distribución del empleo obedece en un mayor sentido a una lógica regida por la presencia de importantes economías a escala en las ciudades capitales, que promueva y refuerza la localización de las empresas y consumidores en dichas ciudades.

No obstante, es posible sugerir la presencia de municipios que representan subcentros económicos regionales. Por ejemplo, en el caso de la región Pacífico Sur (departamento del Valle del Cauca), se destacan municipios como Palmira, Buenaventura, Buga, Tuluá y Cartago y en el Eje Cafetero se tiene el caso de La Dorada, el cual pertenece al departamento de Caldas.

De otro lado, la región Andina norte obtuvo un gradiente de $-0,515 \%$; la región Central arroja un valor de $-0,649 \%$ y la región Caribe un $-0,274 \%$.

En este grupo de regiones, la distribución del empleo en los municipios es menos compacta en torno a las ca-

Tabla 3

Resultados de la estimaciones

\begin{tabular}{lccccccc}
\hline & $(\mathbf{1})$ & $\mathbf{( 2 )}$ & $\mathbf{( 3 )}$ & $\mathbf{( 4 )}$ & $\mathbf{( 5 )}$ & $\mathbf{( 6 )}$ & $\mathbf{( 7 )}$ \\
\hline & Pacífico Norte & Pacífico Sur & Andina Norte & Andina Sur & Central & Eje Cafetero & Caribe \\
\hline$\beta^{0}$ & $9.5560^{* * *}$ & $10.7405^{* * *}$ & $9.9616^{* * *}$ & $9.3765^{* * *}$ & $9.7884^{* * *}$ & $9.9923^{* * *}$ & $9.5681^{* * *}$ \\
& $(50,65)$ & $(20,12)$ & $(33,73)$ & $(41,92)$ & $(36)$ & $(35,94)$ & $(60,88)$ \\
\hline$\beta^{1}$ & $-0,00147$ & $-0,00859^{* *}$ & $-0,00515^{* * *}$ & $-0,00208$ & $-0,00649^{* * *}$ & $-0,00754^{* *}$ & $-0,00274^{* * *}$ \\
& $(-1,75)$ & $(-2,21)$ & $(-3,11)$ & $(-1,13)$ & $(-2,73)$ & $(-2,36)$ & $(-3,44)$ \\
\hline $\mathrm{R}^{2}$ & 0,044 & 0,036 & 0,21 & 0,031 & 0,17 & 0,23 & 0,105 \\
No. de observaciones & 72 & 28 & 50 & 100 & 84 & 34 & 105 \\
\hline
\end{tabular}

Fuente: Elaboración propia.

Nota: entre paréntesis razones t calculadas con errores estándar robustos

${ }^{* * *}$ Significativa al $1 \%{ }^{* *}$ Significativa al $5 \%$.

Figura 1.a

Gradiente Pacífico sur

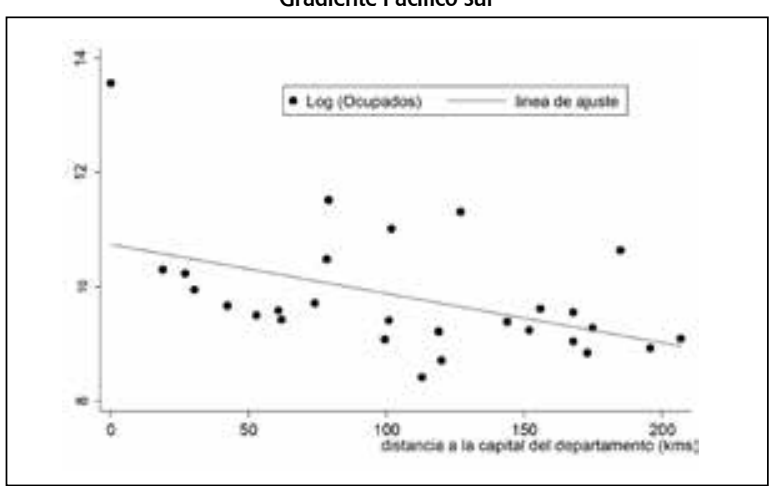

Figura 1.b

Gradiente Eje cafetero

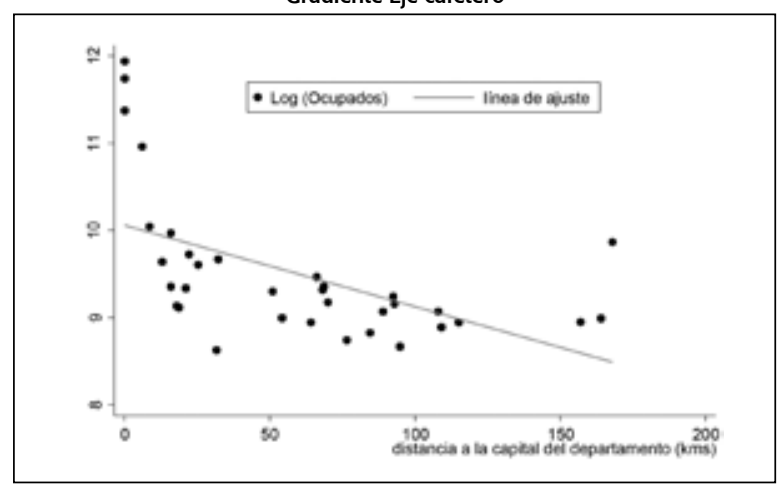

Fuente: Elaboración propia 
pitales; sin embargo, aún se aprecia claramente un patrón de reducción del empleo con el aumento de la distancia a las capitales (Figuras 2.a, 2.b y 2.c). El máximo alejamiento de los municipios sobre las capitales alcanza los 430 kilómetros en la región Caribe.

En la región Andina Norte, además de las capitales (Bucaramanga y Cúcuta), sobresalen algunos municipios que tienen potencial como subcentros: Floridablanca, Barrancabermeja, Girón y Piedecuesta. Por su parte, en la región Central se estructura un esquema de altísima concentración del empleo en la ciudad de Bogotá -capital económica de esta región y del país-y la existencia de una multiplicidad de pequeños municipios que componen los departamentos de Cundinamarca y Boyacá. La región Caribe muestra una distribución espacial de los municipios más dispersa que las regiones anteriores, aspecto que se evidencia al ser la región de mayor extensión y la segunda de menor densidad de empleo por kilómetro cuadrado, dentro de las siete regiones analizadas.

En aquellas regiones donde no es estadísticamente significativo el gradiente (Pacífico Norte y Andina Sur), se mostraría que no hay una distribución exponencial decreciente en los niveles de empleo y por lo tanto, esas regiones presentan economías subregionales o locales con cierta autonomía, autarquía o dependencia de otras áreas diferentes a la ciudad capital. En el caso de la región Pacífico Norte, (departamento de Antioquia), hay un grupo de municipios muy alejados de Medellín con relativamente altos niveles de empleo (Apartadó, Turbo, Chigorodó, El Bagre-Nechí y Caucasia). Así, la distancia y la elevación de los costos de transporte favorece el posicionamiento de estos municipios como subcentros económicos en el departamento de Antioquia (Figura 3.a). En la región Andina Sur (Cauca, Narińo, Tolima y Huila), a partir de las capitales no se aprecia una ordenación de municipios decreciente en el empleo a través de la distancia

Figura 2.a

Gradiente región Andina norte

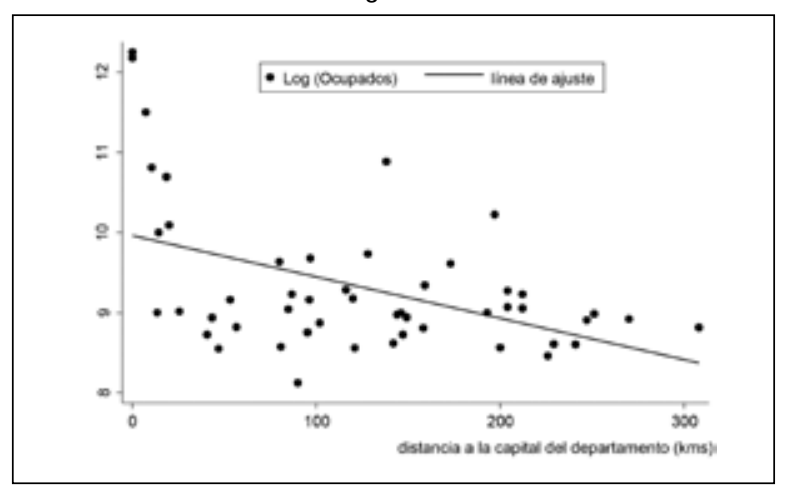

Figura 2.b

Gradiente región Central

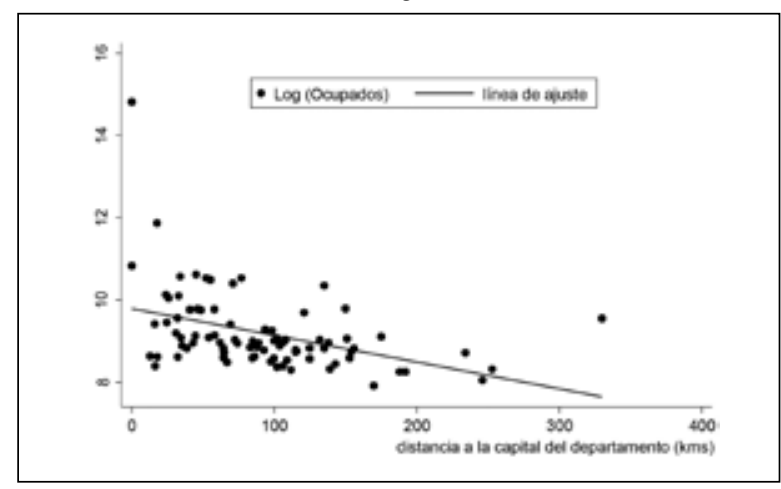

Figura 2.c

Gradiente región Caribe

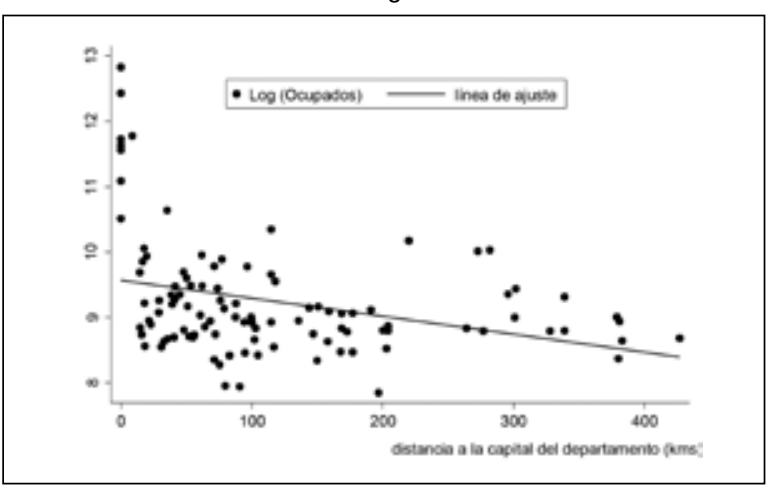

Fuente: Elaboración propia

(Figura 3.b). Además, en los casos de los departamentos de Cauca, Narińo y Huila es notable la ausencia de vías de transporte eficientes que faciliten una adecuada interacción económica entre los municipios y las capitales. Por consiguiente, en la región Andina Sur, factores como la presencia de economías de escala en las capitales no suficientemente fuertes y la ausencia de un sistema de vías de transporte eficiente, posibilitan la existencia de subregiones con dinámicas económicas independientes de las ciudades capitales.

\section{Conclusiones e implicaciones}

En el presente documento se estimaron gradientes de densidad del empleo a nivel intrarregional en Colombia, a través de un modelo log-lineal estimado por M.C.O. Los resultados muestran que en cinco regiones se da una relación negativa y estadísticamente significativa entre los niveles de empleo y la distancia al centro económico.

Las regiones con gradientes significativos fueron Pacífico sur, Eje Cafetero, Andina Norte, Central y Caribe. En estas regiones, la distribución del empleo se ajustó a una forma exponencial decreciente, lo que indica que 
Figura 3.a

Gradiente región Pacífico norte

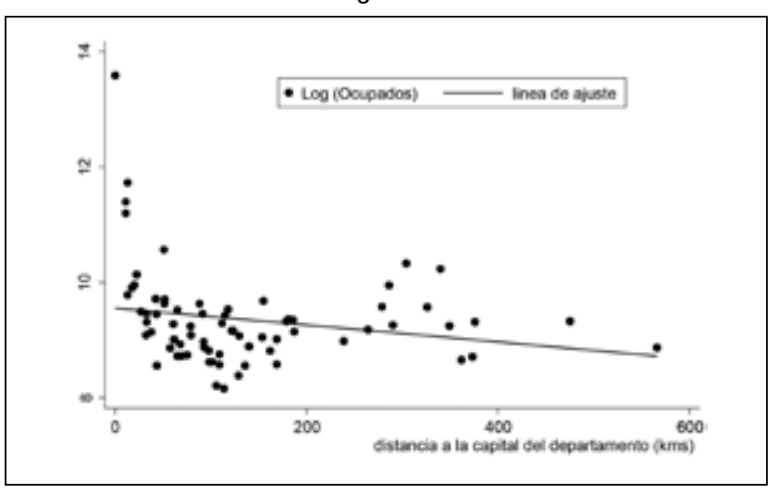

Fuente: Elaboración propia

este tiende a reducirse a medida que el municipio se aleja de la capital. Así mismo, regiones como Pacífico Sur y Eje Cafetero muestran una distribución más compacta del empleo. En ellas, la actividad económica se organiza de acuerdo con una lógica dominada por la presencia de importantes economías de escala en las ciudades capitales. No obstante, es viable sugerir la presencia de municipios que funcionarían como subcentros económicos en estas regiones.

En regiones como Andina Norte y Caribe, aunque la distribución del empleo arroja un gradiente negativo y significativo, esta es menos compacta en torno a las capitales. Por ejemplo, la región Caribe presenta una alta extensión y baja densidad de empleo por kilómetro cuadrado.

La obtención de un gradiente no significativo en regiones como Pacífico norte y Andina sur, revela que la distribución del empleo no se ajusta al patrón sugerido en la ley de Clark, razón por la cual es posible plantear la presencia de economías subregionales con dinámicas autónomas o independientes de las capitales. En el caso de Pacífico Norte, hay municipios muy alejados de la capital Medellín, por lo cual la influencia de las economías que se generan en una ciudad como esta se reduce de manera importante o desaparece hacia los municipios muy alejados, lo que justifica la necesidad de que surjan dinámicas subregionales propias en esos municipios distantes. En lo tocante a la región Andina Sur, los resultados también indican que la distribución del empleo no está influenciada por las economías de escala de las ciudades capitales, ya que la actividad económica de los municipios parece no obedecer a algún tipo de ordenación decreciente a partir de las capitales, aspecto que, además, se ve reforzado por el hecho de que esta región tiene una elevada extensión, la más baja densidad de empleo por kilómetro cuadrado y deficientes redes de transporte. Así, no hay una adecuada
Figura 3.b

Gradiente región Andina sur

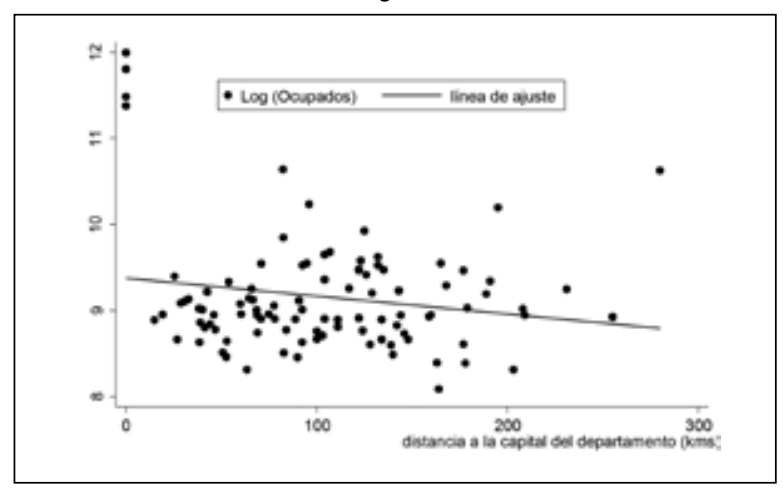

conexión e interacción entre los municipios y las capitales que favoreciera un esquema tipo Clark.

Según el anterior análisis cabría preguntarse cuáles serían las políticas más adecuadas para promover el desarrollo regional: aquellas que favorezcan la concentración de las actividades económicas en torno a las ciudades capitales, con la finalidad de aprovechar las economías de escala tanto externas como internas asociadas a la aglomeración y de esta forma mejorar la competitividad, o aquellas que promuevan el desarrollo de las economías subregionales y con ello reducir las brechas.

El ejercicio aquí planteado constituye una primera aproximación a la problemática de modelar la distribución espacial del empleo en las regiones. Por lo menos a nivel de cinco importantes regiones, se halló evidencia de una distribución tipo Clark. No obstante, el análisis muestra la existencia de subcentros económicos y otorga indicios de dinámicas subregionales autónomas, por lo que sería importante avanzar en ejercicios posteriores e incorporar otras metodologías, como los modelos cubic-spline, los modelos de suavizamiento y las técnicas de la econometría espacial.

\section{Referencias}

Anderson, J. (1982). Cubic-spline urban-density functions. Journal of Urban Economics. 12, 155-167.

Audretsch, D. B., \& Feldman, M. (1996). R\&D spillovers and the geography of Innovation and production. American Economic Review, 86(3), 630-640.

Avendaño, A. J. (2008). Identificación de subcentros de empleo y estimación de funciones de densidad para Bogotá D.C. Recuperado de http://goo.gl/ydCMeP

Bunting, T., Filion, P., \& Priston, H. (2002). Density gradients in Canadian metropolitan regions, 1971-96: Differential 
patterns of central area and suburban growth and change. Urban Studies, 39(13), 2531-2552.

Coffey, W., \& Shearmur, R. (2002). Agglomeration and dispersion of high-order service employment in the Montreal metropolitan region, 1981-96. Urban Studies, 39, 359-378.

Clark, C. (1951). Urban Population Densities. Journal of the Royal Statistical Society, 114(4), 490-496.

Craig, S., \& Ng, P. (2001). Using quantile smoothing splines to identify employment subcenters in a multicentric urban area. Journal of Urban Economics, 49, 100-120.

Dunn, E. S. (1954). The Location of Agricultural Production. Gainesville: The University of Florida Press.

Dunn, E. S. (1955). The Location of Agricultural Production. Journal of Farm Economics, 37(3), 577-579.

Fujita, M., \& Thisse, J. F. (1996). Economics of agglomeration. Centre for Economic Policy Research. Recuperado de http:// goo.gl/UaZ8gF

Glaeser, E. L., Kallal, H., Scheinkman, J. A., \& Shleifer, A. (1992). Growth in cities. Journal of Political Economy, 100(6), 1126-1152.

Glaeser, E. L., \& Kahn, M. E. (2001). Decentralized Employment and the Transformation of the American CityComments (Working paper No. 8117). MA: NBER Working peper series.

Gordon, P., Richardson, H., \& Wong, H. (1986). The distribution of population and employment in a polycentric city: the case of the Angeles. Environment and Planning, 18, 161-173.

Henderson, J. V., Kuncoro, A., \& Turner, M. (1995). Industrial development in cities. Journal of Political Economy, 103(5), 1067-1090.

Jacobs, J. (1975). La economía de las ciudades. Madrid: Península.

Krugman, P. R. (1991). Geography and Trade. Cambridge MA: MIT Press.

Lu, J., \& Guldmann, J. M. (2012). Landscape ecology, landuse structure, and population density: Case study of the Columbus Metropolitan Area. Landscape and Urban Planning, 105, 74-85.

Martori, J. C., \& surinach, J. (2002). Classical models of urban population density. The case of the Barcelona metropolitan area. Nederlandse geografische studies, 303, 109-123.

Mcmillen, D. P. (2004). Employment densities, spatial autocorrelation, and subcenters in large metropolitan areas. Journal of Regional Science, 44(2), 225-243.
Mcmillen, D. P., \& Lester, T. W. (2003). Evolving subcenters: employment and population densities in Chicago, 19702020. Journal of Housing Economics 12, 60-81.

McDonald, J. F. (1987). The identification of urban employment subcenters. Journal of Urban Economics, 21, 242-258.

McDonald, J. F. (1989). Econometric studies of urban population density: A survey. Journal of Urban Economics, 26(3), pp. 361-385.

McDonald, J. F., \& Prather, P. J. (1994). Suburban employment centers: The case of Chicago. Urban Studies, 31, 201-218.

Millward, H., \& Bunting, T. (2008). Patterning in urban population densities: A spatio temporal model compared with Toronto 1971-2001. Environment and Planning A, 40, 283-302.

Muñiz, I., Galindo, A., \& Miguel, A. G. (2003). Cubic Spline Population Density Functions and Satellite City Delimitation: The Case of Barcelona. Urban Studies, 40, 1303-1321.

Mukhopadhyay, A., \& Dutt, A. K. (1994). Population Density Gradient Changes of a Postindustrial City-Cleveland, Ohio (1860-1990). GeoJournal, 34,(4), 515-518.

Ocampo, J. A. (1986). Colombia y la economía mundial 18301910. México: Editorial Siglo XXI.

Polése, M. (1998). Economía urbana y regional: introducción a la relación entre territorio y desarrollo. Costa Rica: Libros Universitarios Regional.

Redfearn, C. L. (2007). The topography of metropolitan employment: Identifying centers of employment in a polycentric urban area. Journal of Urban Economics, 61, 519-541.

RuÍz, N., Roca, J., \& Moix, M. (2012). Análisis de la estructura metropolitana mediante la detección de subcentros de empleo, caso Bogotá. Architecture, City and Environment, 6(18), 297-324.

Sveikauskas, L. A., Gowdy, J., \& Funke, M. (1988). Urban productivity: City size or Industry size?. Journal of Regional Science, 28(2), 185-202.

Von-Thünen, J. H. (1826). Der Isolierte Staat in Beziehung auf Landschaft und Nationalökonomie. Traducción ingles por: C. M. Wartenberg (1966), Von Thünen's Isolated State. Oxford: Pergamon Press.

Veneri, P. (2013). The identification of sub-centres in two Italian metropolitan areas: A functional approach. Cities, 31, 177-185. 